\title{
Understanding Tilt in Esports: A Study on Young League of Legends Players
}

\author{
Minerva $\mathrm{Wu}$ \\ University of California, Irvine, USA \\ minniew@uci.edu
}

\author{
Je Seok Lee \\ University of California, Irvine, USA \\ jesl@uci.edu
}

\author{
Constance Steinkuehler \\ University of California, Irvine, USA \\ const@uci.edu
}

\begin{abstract}
Tilt as a gaming term is associated with frustration, rage, and deterioration of gameplay ability. In this exploratory study, we surveyed 95 esports players in a high school esports league on their definitions of tilt, triggers for tilting, responses to tilt, and perception of its malleability. We found that players are tilted most commonly by their own teammates rather than opponents, with their most negative tilt responses reserved for themselves. The majority surveyed believe that they can change how easily they are tilted and believing so was found to lead players to choose more positive responses to tilt. In contrast, perceiving tilt as malleable was found to increase the probability that participants respond with positive strategies. Implications for efforts to improve esports culture and community are discussed.
\end{abstract}

\section{CCS CONCEPTS}

- Human-centered computing $\rightarrow$ Human computer interaction (HCI); Empirical studies in HCI.

\section{KEYWORDS}

esports, stress, tilt, social-emotional learning

\section{ACM Reference Format:}

Minerva Wu, Je Seok Lee, and Constance Steinkuehler. 2021. Understanding Tilt in Esports: A Study on Young League of Legends Players. In CHI Conference on Human Factors in Computing Systems (CHI '21), May 0813, 2021, Yokohama, Japan. ACM, New York, NY, USA, 9 pages. https: //doi.org/10.1145/3411764.3445143

\section{INTRODUCTION}

Esports can be a toxic space for social interaction; in fact, it is one of the two central problems of esports scholarly work currently (with the other central problem being lack of diversity, which is indeed integrally related) [22]. Under the anonymity of the Internet, intolerant and hateful speech and behavior on the part of players and spectators can poison the metaphorical well for everyone thus engaged; "Communication channels might be abused to harass and verbally assault other players, which negates the very purpose of entertainment games by creating a toxic player-community" [24].

Esports, or electronic sports, are competitive videogame play that include elements of individual achievement, cooperative team play,

\section{(c) (7) (5)}

This work is licensed under a Creative Commons Attribution-NonCommercial International 4.0 License.

CHI '21, May 08-13, 2021, Yokohama, Japan

(C) 2021 Copyright held by the owner/author(s).

ACM ISBN 978-1-4503-8096-6/21/05

https://doi.org/10.1145/3411764.3445143 competition, and entertainment. Esports is a dual player-spectator experience, where the players of the game also participate as spectators of "serious", professional play, and the bulk of spectators also play in some capacity [28]. While motivations for playing and spectating differ, [6] teams are randomly generated and then quickly thrust into a match where their trust in their teammates is immediately tested under pressure [12]. When teamwork works, players support each other, and the relationships built from playing together can bleed out to create supportive emotions and esteem support [11]. However, esports' construction of high competition and short-term interactions among randomly matched players may also be what encourages toxic behavior, with noxious player communication and in-game behavior. Competition exacts skill and concentration that alone distinguishes it from more leisure forms of play [13, 30,33], amplifying stress on players and the pressure to perform. To win, esports players need to regulate their own emotional responses.

Failure to self-regulate while playing can contribute to the perceived toxicity of esports games. One instance of perpetuating toxicity can be referred to as "tilt," a phenomenon in which players are triggered by a person or event in the game which generates frustration and other negative emotions, and in turn starts to negatively impact decision-making and gameplay overall. Other definitions emphasize repeated failures, hard work that does not seem to pay off, or heightened affect that interferes with objective decision-making. While all these definitions lurk in the cultural understanding of tilt, this exploratory study highlights young players' understandings of tilt. An understanding of not only what tilt is, but also how young players understand it will help designers and educators better understand how emotional regulation happens in esports and identify opportunities for pedagogical growth and address an emotion-regulation failure that may be undermining young players' gaming behaviors.

\section{BACKGROUND AND RELATED WORK}

Tilt as a term originated from pinball, where frustrated players would hit the machine; the pinball machine would flash "tilt" and the game would be over [8]. Being "on tilt" is closely associated with gambling and is related to poor money management and selfcontrol while playing. The construct of tilt was adopted by the poker community when Browne used tilt as an explanation for why poker players would continue to play despite heavy losses and make increasingly poor decisions [5]. Widely known in the poker community, tilt is associated with losing control due to negative emotions [31] or even letting any emotion (positive or negative) influence rational decision making [27]. Irrational play patterns due to streakiness can also be found in physical sports. The "hot hand" fallacy is a common misbelief of basketball players and fans that 
a player's probability of scoring a shot is higher after a successful shot than after a failure on the previous shot, regardless of the fact that there is no positive correlation between the result of successive shots [14]. Even though gambling and physical sports require acute mental and physical skills to compete at the professional level, the results are affected by coincidence and probability, which leads players to obtain such perceptions.

As adapted to esports, tilt is described as part of the player experience. Broadly, tilt is an emotional reaction to in-game events that cause a deterioration in gameplay. Tilt in esports is primarily associated with frustration and rage while playing and comes up alongside investigations of toxic or deviant behavior. Like in other sports, it is followed by repetitive play which may lead to losing streaks that negatively impacts player experience and may even disengage players from the game itself [20]. Researchers have highlighted the role of anger and frustration as one form of emotional motivation [4] and designers have looked to facial recognition software [31] and physiological wearables [13] to help detect these emotions. Anger or frustration though, while commonly associated, is only part of tilt: aside from this emotional, affective component, tilt is also tied to the erratic [31], irrational [27], nonstrategic [32] play and decision-making resulting from these emotions.

In Multiplayer Online Battle Arena (MOBA) games such as League of Legends, players are divided into different roles on two teams that work to conquer the opposing team's base. This primary goal of destroying the other team's base is supplemented by individual metrics of performance such as kills on the other team. Prior work in motivations to engage with MOBAs has indicated the importance of social interaction in these games [17] and how player individual motivations and goals approaching the game temper their expectations and what causes them to react aggressively [26].

Previous research on toxicity more broadly in competitive video games has focused on the collaborative player experience and the level of communication that is needed to not only play, but play effectively. Kou and Gui discuss the unique experience of playing with strangers in temporary teams: good communication is necessary in order to be successful not only in a particular game, but in overall self-improvement as a player [19]. Problems in communicationpoor social interactions-can destroy teamwork. More experienced players are able to better adapt their skills and strengths in order to facilitate team cohesion and strategy [18]. Having different strategies available is also important to understanding and managing tilt: players who were highly motivated but also frustrated benefited from a cognitive reappraisal and reframing of the task, while players with low motivation or effort benefited most from simply accepting and acknowledging that they were frustrated [29].

Finally, prior research on failure in gaming has firmly established the importance of mindset in learning from and persisting in the face of failure. People who believe intelligence is a fixed, unchangeable construct are more likely to avoid challenging cognitive tests and less likely to persist as they encounter difficulty; meanwhile, people who believe that intelligence is malleable are more likely to persist in the face of challenge and frustration [10]. These findings have been seen in gaming contexts as well, where people of a malleable or mastery orientation are more willing to persist in the face of failure and frustration and take failures as opportunities for improvement [1].

\section{METHODS}

In order to investigate young players' conceptions of tilt, its causes, and its consequences among youth players, we conducted a mixedmethods study including a survey of youth esports players and interviews with their staff to understand how they defined tilt, what triggered tilt, how players commonly responded to tilt, and whether or not they viewed it as something that could be changed. Our focus on youth players reflects our broader interest in developing youth programs for learning that build on young people's interest.

\subsection{Research Questions}

Our specific research questions were the following:

1. How do players define tilt?

2. What triggers tilt?

3. What responses do players have?

4. Do they view tilt as malleable?

One might reasonably expect that different tilt triggers elicit different responses and that one's beliefs about how fixed versus malleable tilt might shape one's responses to different triggers, be they game, loss, self, or other people. These expectations are represented by the following hypotheses:

\section{H1. Different types of triggers elicit different types of} responses.

Different players have different motivations to play, and these different goals temper their expectations of their gameplay experience. We reasoned that these expectations would cause players to react differently when tilted by different aspects of their experience.

H2. One's beliefs about tilt's malleability determines how one responds to tilt.

We reasoned that players' attitude towards the malleability of tilt could parallel malleable or fixed mindsets and affect how the players responded to tilt.

\subsection{Sample}

Youth esports players were recruited for this study through the North American Scholastic Esports Federation (NASEF), one of the largest youth esports leagues now available for youth in the United States, Canada, Mexico, United Kingdom, France, Israel, and Japan [25]. School-affiliated teams compete in structured leagues both regionally and nationally. The NASEF league was chosen as a site for investigation as one of the larger school-sanctioned youth esports organizations serving over 900 schools in North America. Within this program, trained coaches and sponsoring school-site teachers serve as mentors and facilitators, working with players to improve their performance within the game and on the team. A total of 95 players and 8 staff participated in the investigation, which was closed early due to university shutdown of research during the COVID-19 pandemic. Students were in grades 9-12 and enrolled in their high school's NASEF club. Of students in NASEF who reported their gender, $9 \%$ identified as female, $90.7 \%$ as male, and $0.3 \%$ as nonbinary. At the time of data collection, the competitive game played was Riot Game's League of Legends, a massive online battle 
arena game where two teams of five players each compete in a preset map.

\subsection{Player Survey}

The player survey was conducted online, as part of a longer survey, and included four questions, each representing a separate variable.

1. Tilt Definition: What are some words you associate with tilt? (open response)

2. Tilt Triggers: What gets you tilted? (open response)

3. Tilt Responses: When you feel tilted, what do you do? (open response)

4. Perceptions of Malleability: Do you think you can change how easily you get tilted? (Yes, No, I don't know)

\subsection{Staff Interviews}

As a way to confirm and triangulate patterns found in the player survey data, 8 staff members ( 7 general managers and 1 coach) were interviewed about player tilt behaviors they observed and strategies they use to bolster self-regulation skills. A general manager (GM) was a school teacher at the club sites who took on the role of club GM in addition to their usual instruction. All interviewed GMs took an active role in guiding and helping the club, many helping to coach students in emotional regulation, communication, and analysis. Even though the teacherGMs were not our primary target, they were interviewed as witnesses of player behavior and attitudes. These interviews were conducted as part of a longer interview across a range of subjects; only tilt-related questions will be discussed in this analysis.

All interviews were conducted on Discord (a voice application designed for gaming) by trained, graduate-level researchers, with the tilt-related portion of the interview lasting 20-25 minutes total. Tilt-specific questions mirrored the player survey subjects: Staff members were asked about their own understanding about the tilt phenomenon, their observations of what triggers player tilt and how players frequently responded, and their perception of whether tilt could be changed. While not included on the player survey, one additional question asked staff whether and what strategies staff recommended to improve self-management and mitigate tilt.

\subsection{Data Analysis}

This study used a mixed method approach, beginning with qualitative data and then quantifying those data as needed to examine and measure approaches among nominal variables. Given the exploratory nature of the study, both the player survey data and the staff interview data were coded using codes developed using an inductive or grounded theory [15] approach. That is, coding schemes were determined by reviewing, comparing, and grouping the available data then applying the scheme across all items, rather than imposing preset codes.

For the survey data, two researchers reviewed all response data independently, then met to discuss codes. One researcher applied the codes, and any uncertainties were discussed. Each code was then examined for internal consistency as a check for coding drift and errors. Responses that were indecipherable, ambiguous, or off-topic were categorized as "uncodable" and removed before analysis; three
(3) propositions total were thus removed. Responses containing multiple answers to a given prompt were broken down into separate propositional units before coding, resulting in a data corpus of $\mathrm{n}=124$ propositions total.

All staff interviews were audio-recorded, transcribed and thematically analyzed by three researchers trained in content analysis methods. Researchers picked the first three interview transcripts and analyzed them together using inductive coding to generate an initial coding scheme. Each then individually coded the rest of the three transcripts based on the scheme. All coders discussed and compared the coding results together, with adjustments made after unanimous agreement was reached. The lead analyst then individually coded all remaining data using the finalized scheme.

To test our hypothesized relationships between variables stated (above), we cleaned the data and then tested each relationship using Lambda $(\lambda)$ measure of association between two nominal variables. We also examined relationships among tilt triggers, tilt responses, and malleability of tilt. For these analyses, No tilt responses $(n=4)$ were removed to allow us to test only those responses in which tilt was reportedly present. For the variable tilt trigger, we combined team, opponents, and others into a single new category people to reduce the number of empty cells. Lambda was used to measure the association of each pair of variables, corresponding to our two hypotheses.

\section{RESULTS}

\subsection{Tilt Definitions}

Our first question was to understand how players themselves define tilt. Tilt is a relatively new construct to esports, so our first task in this study was to better understand how youth players in our program conceptualized it. Students were not prompted with a definition of tilt, but asked to provide their own. Of 95 students, 3 students responded "I don't know" and 6 others had indecipherable responses. Figure 1 below gives frequency counts for player responses to the first survey question, "What are some words you associate with tilt?" Presented definitions are, as much as possible, students' own terms: while we collapsed word families such as "anger" and "angry", differences like "mad" and "angry" remained distinct. Responses with a frequency of 1 are omitted.

The emotions anger, frustration, and toxicity are players' most frequent associations with tilt, with similar emotions mad, annoyed, and rage the second most frequent group of associations. Thus, players' definitions are in keeping with the word's current usage within the context of esports, with negative "emotional reaction" at the construct's core. As we go down in response frequency, we see Browne's "deterioration of gameplay" component [5] also enter as both cognitive (cloudy, confused) and behavioral (throwing).

Esports program staff definitions included anger and frustration (Staff 1, 6, 7, 8), a sense of hopelessness (Staff 2, 3, 5) indicated by loss and losing streaks (Staff 2,4) and ties to toxicity. While not expressed by players, staff members also discussed tilt as a deviance from the usual level of gameplay (Staff 4,5). Thus, though associated words and definitions vary, player and staff definitions do keep in line with tilt definitions with how the term has been used in poker and portrayed in esports media. 


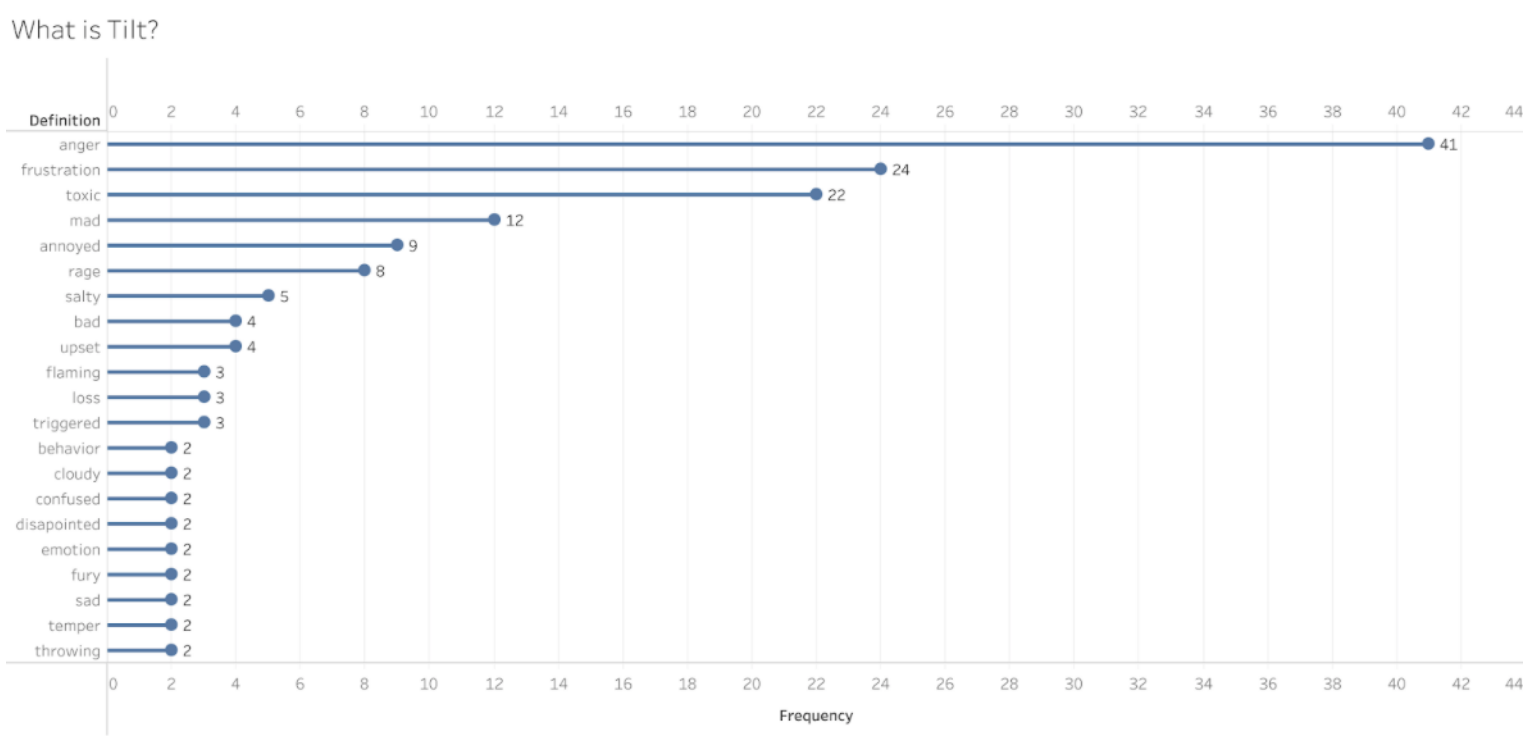

Figure 1: Esports players definitions of tilt.

\subsection{Tilt Triggers}

When players were asked what triggers them to tilt, their most common (modal) answer is their team members (36\% of responses). Answers that fell into this category include examples such as "bad and trolling teammates," "not having a communicative team," and "my team making obvious mistakes". The second most prevalent response (22\%) was other players in general (coded as simply "others"), without specification of their role or relationship. Example answers here include "other people flaming or [badmouthing] me," "toxic players" and people using particular tactics such as "campers" and "spammers." Opponent players, on the other hand, were reported as the tilt trigger only $2 \%$ of the time. Thus, while other players appear to be the primary cause of tilt among players surveyed, it was predominantly not competitive players that tilted players, but collaborative ones.

Beyond the frustrations caused by other players, self was the next most frequently cited tilt trigger. Nearly one-fifth (19\%) of players surveyed reported that frustration or disappointment with their own gameplay performance was a primary tilt factor. Responses in this category include "dying to something that I shouldn't have, or doing something that I know is wrong but still [doing] it," "messing up," and "my own mistakes." Such self-tilt may be reflective of the narrow esports context alone or may well be part of the current stress and anxiety epidemic among teenagers in the US and pressures to excel [16].

Finally, losing (14\% of responses) a match (or a string of matches) and issues with the game itself (3\% of responses) were the lowest reported tilt triggers. Responses categorized as loss include examples such as "losing seemingly easy victories" and "repeat losses." Responses categorized as game include "unbalanced/untested game mechanics" and "when my controller doesn't seem to be responding." Thus, while loss conditions and the game are sometimes the context of tilt episodes, they are not as often seen as triggers for tilting in and of themselves.

\subsection{Tilt Responses}

When players were asked how they responded after tilt was triggered, their most common (modal) answer was to exit the game (32\% of responses). Example responses in this category include "stop playing" and "take a break." Exit does not necessarily mean immediately quitting mid-game, but stopping play after the match has ended and doing something else. Exiting the game is a productive and appropriate strategy, frequently recommended in popular online media as a way to stop tilt, since it resets emotions so players do not take their agitation from one game and act recklessly in the next. Programs designed to detect and lessen tilt emphasize this strategy: the technology helps ameliorate tilt it helps identify when the player is agitated so they know to exit the game and take a break [31i, gilleade]. Browne called exiting the preferred method of break-even poker players: when the player realizes they are tilted, they would take the loss and stop playing after that round, essentially cutting their losses and coming back to the table when they had a clearer mindset [5].

The second most common responses to tilt were also, in fact, positive. One-fourth (25\%) of players, in response to tilting, applied a productive strategy other than simply exiting. Examples include "calm[ing] myself down while focusing on the game," "calm[ing] down," and "try[ing] to smile." Five respondents not only selfregulated in response to tilt triggers but also attempted to learn from it or, in the words of one player, "try and figure out what went wrong."

Yet, nearly the same proportion of players surveyed (22\%) admitted to decidedly negative responses to tilt, including actions such as "scream/ yell" "get mad, flame" and "smash my desk." Thus, tilt remains an area where additional progress can be made. Staff interview data corroborated this, indicating that in-league, there would be instances where players would yell (37\% of staff) or blame (37\%) other things. 


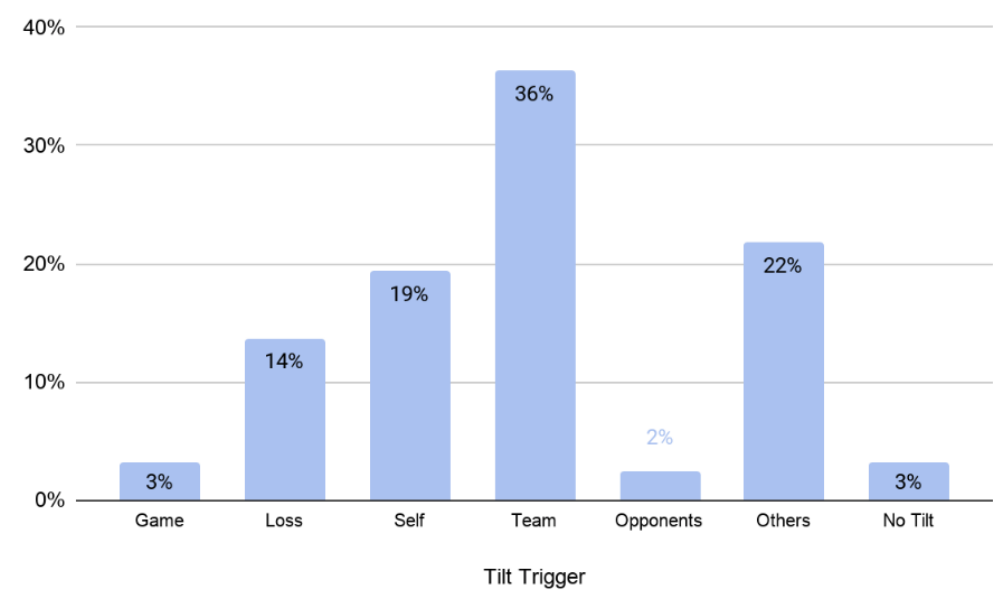

Figure 2: Player self-reported tilt triggers.

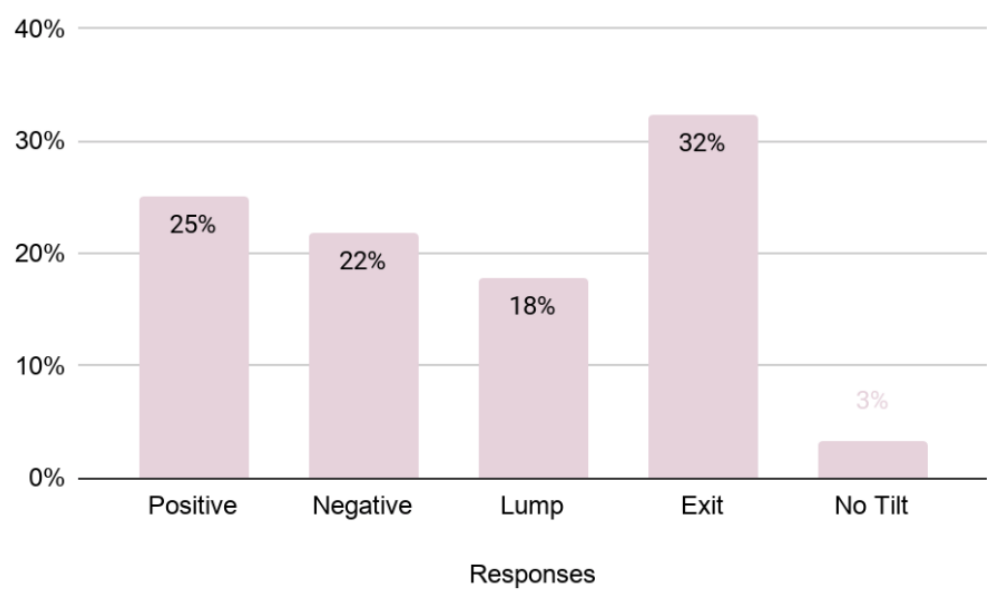

Figure 3: Player self-reported responses to tilt.

The remaining player $18 \%$ of players reporting tilt had neither a positive or negative approach to tilt but simply opted to do nothing. We coded such non-responses as lump, a term drawn from the business literature to refer to employees willing to "take their lumps" and simply accept bad things that happen to them [3]. Example responses include "talk less," "play more games" and "nothing." Lumping is neither good or bad in itself, although simply tolerating triggers does little to address the source or the outcome. Staff interviews offered more insight: lumped behaviors were subdued responses, when the players fell quiet and stopped communicating (Staff 3, 4, 5) or get demoralized and express discouragement (Staff 2,5 ); one GM called this "a sneakier level of tilt, I feel, where they haven't given up, they're not trying, but they're not trying their best either. ...And that's a little bit harder to coach and encourage" (Staff 5, p. 14).

Finally, in interviews, Staff 1, 3, 5, and 6 also noted that their players demonstrated "erratic" behaviors when tilted: a player might be more verbal or stop communicating, and they would play differently (often more poorly). This out-ofcharacter behavior was less about what the player was actually doing and more about a sudden shift in behavior or attitude.

\subsection{Perceptions of Malleability}

Finally, participants were also asked if they thought they could change how easily they get tilted. The modal answer was yes $(67 \%$ of responses), indicating that indeed players see tilt as something malleable and therefore, by inference, able to be improved. Roughly one-fifth $(21 \%)$ of players responded that they do not know whether tilt is changeable. Only $12 \%$ responded no, that tilt is not changed and, by extension, could not be addressed. This belief in malleable versus fixed emotional volatility is, perhaps in some way, the effective equivalent to the notion of fixed versus growth mindset [9]. 


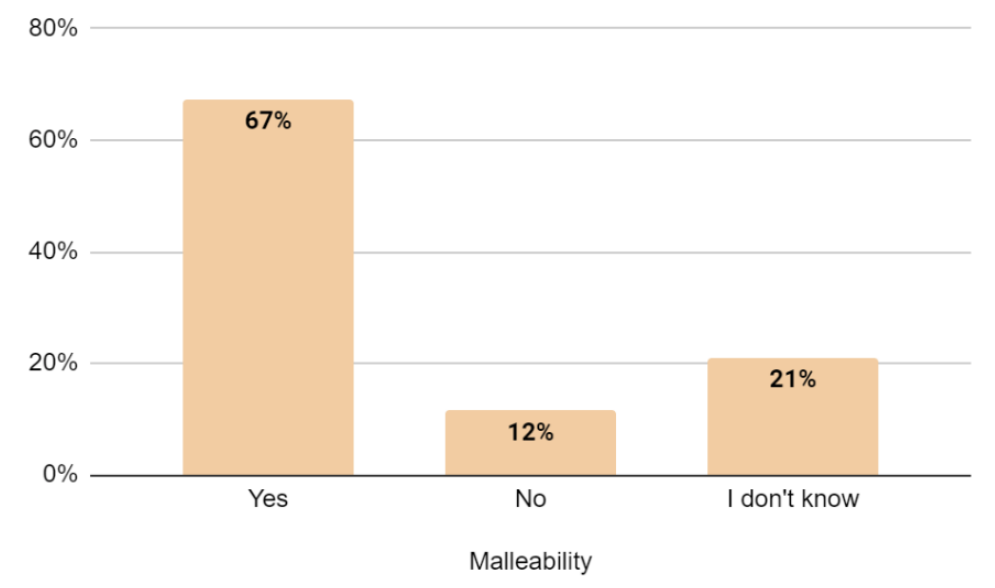

Figure 4: Player perceptions of the malleability of tilt.

Table 1: Each hypothesized relationship tested with resulting lambda and p-value.

\begin{tabular}{lll}
\hline Cause $(I V) \rightarrow$ Effect $(D V)$ & Lambda $(\lambda)$ & P-value \\
\hline Tilt trigger $\rightarrow$ Tilt Response & 0.375 (strong) & 0.000 \\
Perception of Malleability $\rightarrow$ Tilt Response & 0.342 (strong) & 0.000 \\
\hline
\end{tabular}

Table 2: Tilt Response as an Effect of Tilt Trigger

\begin{tabular}{|c|c|c|c|c|c|}
\hline \multicolumn{6}{|c|}{ Tilt Trigger } \\
\hline Tilt Responses & Game & Loss & Self & People & \\
\hline Positive & $25 \%$ & $18 \%$ & $8 \%$ & $33 \%$ & \\
\hline Negative & $0 \%$ & $41 \%$ & $42 \%$ & $13 \%$ & \\
\hline Lump & $0 \%$ & $6 \%$ & $8 \%$ & $25 \%$ & \\
\hline Exit & $75 \%$ & $35 \%$ & $42 \%$ & $28 \%$ & \\
\hline \# of responses & 4 & 17 & 24 & 75 & 120 \\
\hline
\end{tabular}

\subsection{H1. Different types of triggers elicit different types of responses.}

Lambda was calculated for the impact of tilt trigger on tilt response, and for perception of malleability on tilt response. Lambda values range from 0.0 (no relationship) to 1.0 (perfect relationship) with weak associations ranging from $0.01-0.09$, moderate associations ranging from $0.10-0.29$, and strong associations ranging from 0.30 -0.99 [2]. Resulting test statistics and p-value for each relationship are shown in Table 1 below. Both relationships are significant ( $\mathrm{p}$ values $<0.05)$ and strong $(\lambda>0.30)$.

Our data show that, indeed, different tilt triggers lead to different strategies. As shown in Table 2 below, the predominant response when the game triggers tilt is to simply exit (75\%). The predominant response when a loss triggers tilt is either simply exiting (35\%) or overtly negative (yelling, throwing things, etc) $(41 \%)$. When one's own self-performance triggers tilt, we see more variation in responses but simply exiting and overtly negative responses equally dominate ( $42 \%$ and $42 \%$ respectively). But when the tilt trigger is other people, the predominant response shifts to positive (33\%), followed by simply exiting (28\%), putting up with it (lump) (25\%), and only lastly acting out (negative response) (13\%). Based on these data, it seems that players' harshest responses are reserved for oneself while one's best efforts at containing the frustration are reserved for other people.

\subsection{H2. Beliefs about tilt's malleability determine how one responds to tilt.}

As to the second hypothesis, our data show that indeed one's beliefs about whether or not tilt is malleable determine how one responds to it. As shown in Table 3 below, different perceptions of malleability lead to different tilt responses. Players who believe tilt malleable reported positive tilt responses more than players who believed otherwise ( $31 \%$ positive versus $0 \%$ negative and $15 \%$ uncertain). Players who believed tilt is fixed reported negative tilt responses more than other players (36\% versus $27 \%$ and $27 \%$ respectively). Players chose lumping and exit strategies roughly equally, 
Table 3: Tilt Response as an Effect of Perceptions of Tilt Malleability

\begin{tabular}{|c|c|c|c|c|}
\hline \multicolumn{5}{|c|}{ Perceptions of Malleability } \\
\hline Tilt Responses & Yes (Malleable) & No (Not Malleable) & I Don't Know & \\
\hline Positive & $31 \%$ & $0 \%$ & $15 \%$ & \\
\hline Negative & $18 \%$ & $36 \%$ & $27 \%$ & \\
\hline Lump & $20 \%$ & $27 \%$ & $15 \%$ & \\
\hline Exit & $31 \%$ & $27 \%$ & $38 \%$ & \\
\hline \# of responses & 87 & 8 & 25 & 120 \\
\hline
\end{tabular}

Table 4: Tilt Trigger effect on Tilt Response under each of Levels of Perceptions of Tilt Malleability

\begin{tabular}{|c|c|c|c|c|c|c|c|c|c|c|c|c|c|c|}
\hline \multicolumn{5}{|c|}{ Tilt is Malleable } & \multicolumn{5}{|c|}{ Tilt is Fixed } & \multicolumn{5}{|c|}{ I Don't Know } \\
\hline $\begin{array}{l}\text { Tilt Re- } \\
\text { sponse }\end{array}$ & Game & Loss & Self & People & $\begin{array}{c}\text { Tilt } \\
\text { Re- } \\
\text { sponse }\end{array}$ & Game & Loss & Self & People & $\begin{array}{c}\text { Tilt } \\
\text { Re- } \\
\text { sponse }\end{array}$ & Game & Loss & Self & People \\
\hline+ & $50 \%$ & $27 \%$ & $11 \%$ & $38 \%$ & + & $0 \%$ & $0 \%$ & $0 \%$ & $0 \%$ & + & $0 \%$ & $0 \%$ & $0 \%$ & $31 \%$ \\
\hline- & $0 \%$ & $27 \%$ & $39 \%$ & $11 \%$ & - & $0 \%$ & $50 \%$ & $0 \%$ & $50 \%$ & - & $0 \%$ & $75 \%$ & $50 \%$ & $8 \%$ \\
\hline Lump & $0 \%$ & $9 \%$ & $11 \%$ & $25 \%$ & Lump & $0 \%$ & $0 \%$ & $0 \%$ & $17 \%$ & Lump & $0 \%$ & $0 \%$ & $0 \%$ & $31 \%$ \\
\hline Exit & $50 \%$ & $36 \%$ & $39 \%$ & $27 \%$ & Exit & $0 \%$ & $50 \%$ & $0 \%$ & $33 \%$ & Exit & $100 \%$ & $25 \%$ & $50 \%$ & $31 \%$ \\
\hline & 2 & 11 & 18 & 56 & & 0 & 2 & 0 & 6 & & 2 & 4 & 6 & 13 \\
\hline
\end{tabular}

regardless of their beliefs about tilt. Therefore, we conclude that perceiving tilt as malleable has a positive effect on how players respond when tilted, leading them to choose more positive responses to tilt overall.

\subsection{Relationships Among All 3 Variables}

In order to examine the interrelationships among all three variables, we constructed partial tables of the zero-order relationship "Tilt Trigger on Tilt Response" (shown in Table 2) within each level of the test variable Perceptions of Malleability - in effect, holding our test variable constant to examine the relationship between tilt trigger and tilt response at each level of the test variable: belief that tilt is malleable, believe that tilt is not malleable, and uncertainty (ie. I don't know). Finally, we examine the relationships of all three variables. The partial tables are shown in Table 4 below. Please note that due to several cell counts of zero, lambda is not as useful.

Examination of the partial tables reveals an important pattern: Perceptions of malleability specify the conditions under which different tilt triggers result in different tilt responses. If a player believes that tilt is malleable, then responses shift toward positive and exiting when the game or a match loss is triggering them. In other words, perceiving tilt as malleable amplifies positive responses, even when the trigger cannot feel hurt or harm from what the player does. If the player believes that tilt is fixed, then strategies shift from tilt responses that avoid abusiveness (here, positive responses, lumping, and exit strategies) to negative responses and exiting when it's other people triggering them. In other words, perceiving tilt as fixed seems to amplify negative strategies toward others, perhaps resulting in a kind of "not my fault if I hurt you, I can't help it" attitude. When the player themselves are the trigger, however, their beliefs about the malleability of tilt make no difference in how they respond. More productive beliefs about tilt's malleability do not protect players from the likelihood of negative responsiveness, self-talk, and reactivity.

\section{DISCUSSION}

These findings remain preliminary but still contain implications for program development and intervention for youth. First, players are tilted most commonly by their own teammates, suggesting that interventions designed to target and promote team communication and collaboration skills may be one potent way to address and potentially ameliorate tilt triggers themselves. Such skills extend beyond competitive gaming, yet competitive gaming offers a key arena for their development. As one GM interviewed for this study recognized, "collaboration is a huge part of [communication]. That's one big thing that we don't always get to address in the classroom...but a majority of the games that are out there for esports - it is a huge team effort to get that tournament win" (Staff 7 , p.13). Already HCI designers are working to improve virtual teammates' awareness and trust of each other as a way of facilitating communication [12].

Second, players are also heavily tilted by their own performances. In fact, based on these data, it seems that players' harshest responses are reserved for themselves while their best efforts at containing the frustration are reserved for other people. Players dedicate a lot of time and hard work into improving at the game; their frustration and even moral anger is unfortunately understandable if they feel like their efforts have not been rewarded [32]. Given the current problem of stress and anxiety among high school teenagers in the US and reported pressure to excel [16], tilt in response to one's own actions and performance may offer teachable moments through which GMs and Coaches might remediate self-judgment, negative self-talk, and stress. Staff interviews illustrated how GMs or coaches might reframe players' approach to tilt and dealing with loss or 
frustration in game: they would focus on preventing tilt from even occurring (Staff 1, 2, 3, 6, 8), usually by by reframing goals of the game as constantly improving instead of just winning. For strategies when tilt does arrive, players are encouraged to recenter their emotions (Staff 1, 5, 6, 8) and shift their attitude towards what they can control (Staff 2, 3, 5). They discuss what better communication looks like despite setbacks $(1,3,5,8)$ and emphasize the moment of tilt as an opportunity to learn from $(2,3,5)$. Esports and education offers opportunities for addressing this self-reproach: efforts targeting wellness and self-care in these areas hold some promise in addressing toxicity online by first addressing toxicity toward oneself.

Regardless, negative responses to tilt - "getting toxic and smashing my desk," "slamming my fist on the table, sometimes slamming my mouse," "getting angry and frustrated at the game / myself," "ranting and get mad," "critiquing my friends when I spectate," "flaming," "screaming," "yelling a lot and hitting things", "cussing people out," "blaming others," - are still almost as common as positive responses, so there is much work left to do in changing interactions with others. In the words of one GM interviewed, this is another opportunity for esports in education:

A lot of our kids haven't been coached in any kind of activity before so they don't really know how to be good losers because they've been brought up by the internet and they tend to be toxic when they start losing or even when they're winning. And I think esports...especially esports clubs in high school and middle school, can really help teach gamers how to game correctly and game properly and be good citizens in the world rather than just having them learn from the other toxic people on the internet." (Staff 8 , p. 15)

Fortunately, most players do believe that whether and how significantly one gets tilted can be changed. Our evidence suggests that perceiving tilt as malleable has a positive effect on how players respond when tilted, leading them to choose more positive responses to tilt overall. Perceiving tilt as fixed, however, has the opposite effect, tamping down productive responses to tilt and amping up more negative and abusive strategies toward other players who perhaps triggered it. Belief in malleable versus fixed emotional volatility is somewhat similar to the notion of fixed versus growth mindset [9]: How you view tilt's changeability may predict how well you hold yourself accountable to your reactions online. Just as interventions designed to teach students about growth mindset help with persistence in the face of challenge [10], $t$ interventions by mentors and coaches to reframe players' perceptions of tilt could encourage more positive responses.

Tilt is an important construct to understand because it represents the esports version of self-management, a core social-emotional skill and necessarily to dealing with everyday tasks and challenges in school and beyond [7]. Analyzing the emotive factors of emotional experiences from League of Legends, Kou and Gui also identified achievement, teammate, game design, and social-identityrelated situations, which parallel our loss, team, game, and self trigger codes, respectively [21]. Their emotion regulation strategies were brought up by our coaches: for example, exiting if tilted is an example of situation selection, reframing game goals is cognitive change, and shifting attitudes in came is attentional deployment. The "emotion work" essential to winning in-game [5] is the emotional regulation and self-management demanded of social interactions beyond the game. Managing tilt resonates with players and they may be motivated to address tilt given its negative impact on peers and play. With the right intervention, players have a unique opportunity to develop their ability to self-regulate their emotions and attention alongside their communication and language skills [23]. Unlike most naturalistic contexts for videogame play, esports provides a structured context in which emotional volatility and lack of self-control can be directly tied to player performance, measured in win or loss. Moreover, as with traditional sports, esports contexts typically include coaches and other mentor roles that offer a natural opportunity for direct intervention by more experienced and mature adults.

\section{LIMITATIONS}

Although our results offer insight into player perceptions of the concept of tilt as well as what triggers them and how they respond, there are some caveats. Firstly of note, the sampled players are part of a school-sponsored league structure, where their participation is still subject to school standards of decorum. The sampled population appears to be overwhelmingly male, which reflects the youth esports player population examined in this study but not the population of game players more broadly. While it is possible that responses to tilt differ by gender, we do not have sufficient data at this time to test this hypothesis. Here, future research is needed. Despite the similarities in inductively generated codes between our high school students and Kou and Gui's general population of League players, we note all student participants were in high school, and children differ from adults in emotional response and regulation [20]. Furthermore, these results reflect what the players reported to in a brief survey, captured at a single point in time; while we had staff observations and insights to corroborate, the data could be bolstered by conversations with the players themselves: such conversations would allow the players to report on their own experiences in a more flexible format than just survey responses. NASEF has also grown, and the data could be supported by a larger sample of players.

\section{CONCLUSION}

Using qualitative survey data from student players, corroborated with interviews with teacher general managers, we reported on high school students' definitions of tilt, triggers for tilting, responses to tilt, and perception of its malleability. Coaching efforts for tilt can provide young players with strategies to enhance their communication and collaboration, thereby mollifying tilt triggers from within the team. Further, GMs and coaches can serve as positive adult mentors who not only discourage negative responses like lashing out, but also help improve students' ability to do emotional work and when stressed or frustrated.

Future work on tilt could additionally consider individual player skills, experience, and familiarity with team members, as different levels of experience may affect player perceptions or motivations in playing. Studies focusing on different esports games-or even non 
esports videogame titles- could help generalize findings. While our exploratory work has drawn associations between triggers and responses, gameplay observations of specific triggers causing certain responses would provide richer evidence for how players respond to in-game stressors, or how out-of-game contexts can influence ingame moods. New wearable technologies could allow us to tie tilt to physiological markers for arousal or frustration, such as blood pressure, heart rate, and skin conductivity [13]. Finally, intervention studies could help identify the best way to scaffold or present emotional management strategies to young players. We look forward to reading more on psychological research in anger, frustration, and emotional management. It is our hope that, by understanding tilt in esports spaces, we can contribute to the broader research effort to understand and possibly ameliorate toxicity online.

\section{ACKNOWLEDGMENTS}

This work was conducted with support from the Samueli Foundation, although the views expressed herein may not reflect the Foundation. We also thank our study participants for their time and insight and the anonymous reviewers for their insightful feedback and guidance.

\section{REFERENCES}

[1] Craig Anderson, Kathryn Campbell, and Constance Steinkuehler. 2019. Building persistence through failure: the role of challenge in video games. In Proceedings of the 14th International Conference on the Foundations of Digital Games (pp. 1-6).

[2] Earl Babbie, Wagner E. William III, and Jeanne Zaino. 2019. Adventures in Social Research: Data Analysis Using IBM SPSS Statistics, 10th Edition. Thousand Oaks CA: Sage.

[3] Steven E. Barkan. 2015. Law and Dispute Resolution. Law and Society: An Introduction. Routledge.

[4] Pierpaolo Battigalli, Martin Dufwenberg, and Alec Smith. 2015. Frustration and anger in games. CES Ifo Working Paper, No. 5258. Center for Economic Studies and ifo Institute, Munich.

[5] Basil R. Browne. 1989. Going on tilt: Frequent poker players and control. Journal of Gambling Behavior, 5, 1, 3-21

[6] Gifford Cheung and Jeff Huang. 2011. Starcraft from the stands: understanding the game spectator. In Proceedings of the SIGCHI Conference on Human Factors in Computing Systems. 763-772.

[7] Collaborative for Academic, Social and Emotional Learning. 2020. Core SEL Competencies. Casel.org. Retrieved from https://casel.org/core-competencies/.

[8] Aaron M. Duncan. 2015. Gambling with the Myth of the American Dream. Routledge Research in Sport Culture and Society. Routledge.

[9] Carol S. Dweck. 1975. The role of expectations and attributions in the alleviation of learned helplessness. Journal of Personality and Social Psychology, 31, 674-680.

[10] Carol S. Dweck, and Ellen L. Leggett. 1988. A social-cognitive approach to motivation and personality. Psychological review, 95(2), 256.

[11] Guo Freeman and Donghee Yvette Wohn. 2017. Social support in eSports: building emotional and esteem support from instrumental support interactions in a highly competitive environment. In Proceedings of the Annual Symposium on Computer-Human Interaction in Play. 435-447.

[12] Guo Freeman and Donghee Yvette Wohn. 2018. Understanding eSports Team Formation and Coordination. Comput. Support. Coop. Work CSCW An Int. J. 27, 3-6 (December 2018), 1019-1050. DOI: https://doi.org/10.1007/s10606-017-9299-4

[13] Kiel M Gilleade and Alan Dix. 2004. Using frustration in the design of adaptive videogames. In Proceedings of the 2004 ACM SIGCHI International Conference on Advances in computer entertainment technology (ACE '04). Association for Computing Machinery, New York, NY, USA, 228-232. DOI: https://doi.org/10. 1145/1067343.1067372

[14] Thomas Gilovich, Robert Vallone, and Amos Tversky. 1985. The hot hand in basketball: On the misperception of random sequences. Cognitive Psychology 17, 3: 295 - 314

[15] Barney G. Glaser, and Anselm L. Strauss. 1967. The discovery of grounded theory: Strategies for qualitative research. Chicago: Aldine.

[16] Juliana M. Horowitz and Nikki Graf. 2019. Most US Teens See Anxiety and Depression as a Major Problem Among Their Peers. PEW Research Center. Retrieved March 2019 from https://www.pewsocialtrends.org/2019/02/20/most-u-s-teenssee-anxiety-and-depression-as-a-major-problem-among-their-peers/

[17] Daniel Johnson, Lennart E. Nacke, and Peta Wyeth. 2015. All about that base: differing player experiences in video game genres and the unique case of moba games. In Proceedings of the 33rd Annual ACM Conference on Human Factors in Computing Systems, 2265-2274.

[18] Young Ji Kim, David Engel, Anita Williams Woolley, Jeffrey Yu-Ting Lin, Naomi McArthur, and Thomas W. Malone. 2017. What makes a strong team? Using collective intelligence to predict team performance in League of Legends. In Proceedings of the 2017 ACM Conference on Computer Supported Cooperative Work and Social Computing, 2316-2329.

[19] Yubo Kou, and Xinning Gui. 2014. Playing with strangers: understanding temporary teams in League of Legends. In Proceedings of the first ACM SIGCHI annual symposium on Computer-human interaction in play, 161-169.

[20] Yubo Kou, Yao Li, Xinning Gui, and Eli Suzuki-Gill. 2018. Playing with Streakiness in Online Games: How Players Perceive and React to Winning and Losing Streaks in League of Legends. In Proceedings of the 2018 CHI Conference on Human Factors in Computing Systems, 1-14.

[21] Yubo Kou and Xinning Gui. 2020. Emotion Regulation in eSports Gaming: A Qualitative Study of League of Legends. In Proceedings of the ACM on HumanComputer Interaction, Vol 4, No. CSCW2, Article 158 (October 2020). 26 pages. https://doi.org/10.1145/3415229

[22] Haewoon Kwak, Jeremy Blackburn, and Seungyeop Han. 2015. Exploring Cyberbullying and Other Toxic Behavior in team Competition Online Games. In Proceedings of the $2015 \mathrm{CHI}$ Conference on Human Factors in Computing Systems, $3739-3748$.

[23] Je Seok Lee, Minerva Wu, Diana Lee, Lee Fleming, Lindsay Ruben, Tom Turner, Kevin Brown, and Constance Steinkuehler. 2020. Designing an Interest-Based Integrated Curriculum Around Esports. International Journal of Designs for Learning. 11, 3 (Aug. 2020), 78-95. DOI: https://doi.org/10.14434/ijdl.v11i3.27663.

[24] Marcus Märtens, Siqi Shen, Alexandru Iosup, and Gernando Kuipers. 2015. Toxicity Detection in Multiplayer Online Games. 2015 International Workshop on Network and Systems Support for Games (NetGames). IEEE. 1-6.

[25] North America Scholastic Esports Federation (2020). "Locations "Retrieved March 2020 from https://www.nasef.org/clubs/locations/

[26] Andrew K. Przybylski, C. Scott Rigby, and Richard M. Ryan. 2010. A motivational model of video game engagement. Review of general psychology, 14(2), 154-166.

[27] Natasha Dow Schull. 2016. Abiding chance: Online poker and the software of self-discipline. Public Culture. 28 (3). 563-592.

[28] Yuri Seo, and Sang-uk Jung. 2016. Beyond solitary play in computer games: The social practices of eSports. Journal of Consumer Culture, 16(3), 635-655. https://doi.org/10.1177/1469540514553711

[29] Catherine A. Spann, Valerie J. Shute, Seyedahmad Rahimi, and Sidney K. D’Mello. 2019. The productive role of cognitive reappraisal in regulating affect during game-based learning. Computers in Human Behavior, 100, 358-369.

[30] Michael G. Wagner. 2006. On the Scientific Relevance of eSports. In International conference on internet computing, 437-442.

[31] Xingjie Wei*, Jussi Palomaki*, Jeff Yan, and Peter Robinson. 2016. The Science and detection of tilting. In Proceedings of the 2016 ACM on International Conference on Multimedia Retrieval, 79-86.

[32] Alfonso White and Daniela M. Romano. 2020. Scalable Psychological Momentum Forecasting in Esports. In SUM '20: State-based User Modelling. 10.13140/RG.2.2.21224.21769

[33] Emma Witkowski. 2012. On the digital playing field: How we "do sport" with networked computer games. Games and Culture, 7(5), 349-374. 